\title{
Changes in Platelet Indices during Pregnancy as Potential Markers for Prediction of Preeclampsia Development
}

\author{
Ahmed Mohamed Nooh ${ }^{*}$, Hussein Mohammed Abdeldayem² \\ ${ }^{1}$ Obstetrics and Gynecology Department, Students' Hospital, Zagazig University, Zagazig, Egypt \\ ${ }^{2}$ Obstetrics and Gynecology Department, Faculty of Medicine, Zagazig University, Zagazig, Egypt \\ Email: ahmed.nooh@zu.edu.eg
}

Received 19 September 2015; accepted 13 October 2015; published 16 October 2015

Copyright (C) 2015 by authors and Scientific Research Publishing Inc.

This work is licensed under the Creative Commons Attribution International License (CC BY).

http://creativecommons.org/licenses/by/4.0/

(c) (i) Open Access

\begin{abstract}
Objective: To assess whether changes in platelet indices, detectable by simple complete blood count (CBC), during pregnancy could be used as markers for prediction of development of preeclampsia (PE). Methods: A total of 2813 pregnant women who received regular antenatal care until delivery were included. Participants were divided into 3 groups: normotensive pregnant women $(n=2621)$, women with PE without severe features $(n=169)$, and women with PE with severe features $(n=$ 23). Blood samples were collected during antenatal visits and/or during the period of in-patient hospital stay, and changes in platelet indices were compared among the three groups. Results: Platelet count (PC) was decreasing while mean platelet volume (MPV) and platelet distribution width (PDW) were increasing as PE progressed. Receiver operating characteristics (ROC) curve analysis showed that PDW had the largest area under curve (AUC) [0.980 (95\% CI: $0.964-1.000)]$, making it the best marker for predicting development of PE. Also, PDW showed the most statistically significant correlation with mean arterial pressure (MAP) $(r=0.902, p=0.000)$, making it the best marker for predicting severity of hypertension. Conclusion: This study provides evidence that PC decreases while MPV and PDW increase as pregnancy advances, and these changes are more pronounced in PE than normotensive pregnancy. These changes predate development of PE by 2 8 weeks and are proportional to the progress of this disorder. The selected platelet indices, especially PDW, have the potential to be utilized as markers for not only prediction of PE development but also severity of hypertension.
\end{abstract}

\section{Keywords}

Platelet Indices, Platelet Count, Mean Platelet Volume, Platelet Distribution Width, Preeclampsia

\footnotetext{
${ }^{*}$ Corresponding author.
} 


\section{Introduction}

Preeclampsia (PE) is a pregnancy-specific multisystem disorder characterized by abnormal vascular response to placentation which is associated with increased systemic vascular resistance, enhanced platelet aggregation, activation of the coagulation system, and endothelial cell dysfunction with resultant reduced organ perfusion [1] [2]. Despite extensive research, the cause of PE remains elusive [3].

Current criteria for a diagnosis of PE require the presence of de novo hypertension (blood pressure of $\geq 140 / 90$ $\mathrm{mmHg}$ ), with proteinuria or any of the other multisystem abnormalities, with onset after the $20^{\text {th }}$ week of gestation [2] [4]-[6].

In modern obstetrics, identification of pregnant women with an increased PE risk is an objective of paramount importance. Recognition of sensitive, specific, cost-effective, and easy to perform biomarkers would allow not only detection of women at risk of PE, but it would also allow a close surveillance, a precise PE diagnosis and a timely intervention. Because PE can progress rapidly, it requires prompt intervention that may include observation in a tertiary care setting and termination of pregnancy, either by inducing labor or by Cesarean section, which is the only known cure for this condition [5]-[8]. As it affects multiple organs, no single, specific and cost-effective marker to predict PE has yet been proposed [9]. However, several models have been suggested but are found to be not clinically relevant [10]-[12].

Platelet indices [platelet count (PC), mean platelet volume (MPV) and platelet distribution width (PDW)] constitute part of the data detectable by complete blood count (CBC) test. Applicability of these indices for the clinical and pathophysiological understanding of vascular diseases, including PE, has been investigated but their value has not yet been fully substantiated [13] [14].

A decreasing PC is observed during the progression of PE, and is suggested to be a characteristic of worsening PE [9]. This PC decline returns rapidly to its normal range after delivery. It has also been noted that MPV increases during pregnancy, and is higher in women with PE. Increased MPV occurs before onset of PE symptoms. Therefore, it may be a valuable marker for development of PE [13]-[15]. Furthermore, it has been suggested that PDW can be a practical tool to evaluate activation of coagulation or thrombocytosis-related disease [16].

The aim of this study was, therefore, to assess whether changes in platelet indices, detectable on CBC during pregnancy could be used as markers for prediction of development of PE.

\section{Subjects and Methods}

\subsection{Study Design}

This was an observational longitudinal study of women attending antenatal clinic (ANC) and/or admitted to maternity ward at Zagazig University Hospital (ZUH), Zagazig, Egypt over the period from $2^{\text {nd }}$ June 2014 to $28^{\text {th }}$ May 2015. The study protocol was approved by ZUH Ethics Committee. Participating women were counselled, a clear explanation of the intervention was given, and a written informed consent was then obtained from all of them.

\subsection{Inclusion Criteria}

Healthy normotensive pregnant women at $\geq 20$ weeks gestation with a live singleton fetus were eligible and approached for recruitment.

\subsection{Exclusion Criteria}

Women with the following conditions were ineligible and excluded: multiple pregnancy, morbid obesity [body mass index $(\mathrm{BMI}) \geq 40 \mathrm{Kg} / \mathrm{m}^{2}$ ], intra-uterine fetal death (IUFD), poor past obstetric history [recurrent miscarriage, $\mathrm{PE}$, pre-term labor, intrauterine growth restriction (IUGR - estimated fetal weight $(\mathrm{EFW})<10^{\text {th }}$ percentile for gestational age), IUFD, or neo-natal death], or currently suffering from a systemic disease (hypertension, gestational or insulin-dependent diabetes, heart disease, renal or hepatic dysfunction).

\subsection{Intervention}

During the enrolment ANC visit at $\geq 20$ weeks gestation, past medical, surgical and obstetric history was re- 
viewed, general and abdominal examinations were carried out, fetal heart rate was checked, and findings were recorded. Routine investigations, including $\mathrm{CBC}$ and urinalysis, were arranged.

In women with prior regular periods, gestational age was estimated from the date of last menstrual period. Otherwise, gestational age was estimated from early ultrasound scan (USS) at 7 weeks gestation. Gestational age was verified by the $13^{\text {th }}$ week USS. Fetal anomaly USS was arranged at around 20 weeks' gestation.

Normotensive women were then reviewed as per the routine antenatal clinic protocol where they attended every 4 weeks until 28 weeks gestation, every 2 weeks until 36 weeks gestation then weekly thereafter until delivery. Those who developed PE without severe features were seen more often while participants who showed severe features of PE were admitted to the maternity ward.

PE was defined as a blood pressure of $\geq 140 / 90 \mathrm{mmHg}$, with proteinuria ( $\geq 300 \mathrm{mg} / 24$ hours urine collection, or $\geq 1+$ on a urine dipstick), or any of the severe features of PE at $\geq 20$ weeks gestation. Severe features of PE included any of the following findings: hypertension: systolic $\geq 160$ or diastolic $\geq 110$ on two occasions at least 4 hours apart while the patient is on bed rest (unless antihypertensive therapy is initiated before this time), newonset cerebral or visual disturbances, pulmonary edema, thrombocytopenia ( $\mathrm{PC}<100 \times 10^{3} / \mathrm{mm}^{3}$ ), impaired liver function (elevated blood levels of liver transaminases to twice the normal concentration), severe persistent right upper quadrant or epigastric pain unresponsive to medication and not accounted for by alternative diagnoses, or both, new development of renal insufficiency (elevated serum creatinine greater than $1.1 \mathrm{mg} / \mathrm{dL}$, or doubling of serum creatinine in the absence of other renal disease) [2] [4]-[6] [17]. The mean arterial pressure (MAP) was calculated using the following formula: $\mathrm{MAP}=[(2$ diastolic blood pressure $)+$ systolic blood pressure $] / 3$ [2] [9].

In normotensive pregnancy and in $\mathrm{PE}$ without severe features, a blood sample for $\mathrm{CBC}$ was taken at each clinic visit, while in PE with severe features; $\mathrm{CBC}$ was performed once or, sometimes, twice daily. Using EDTA samples tubes $(5 \mathrm{~mL})$, venous blood samples were drawn from all participants. Samples were processed within two hours after venepuncture. Automated blood counter Cell-Dyn, 4000 (Abbott Diagnostics, Santa Clara, CA, USA) was used, after calibration, to assess CBC parameters including platelet indices (PC, MPV and PDW).

The primary outcome was to determine the correlation - if any, between changes in selected platelet indices during pregnancy on one hand and development of PE on the other hand.

\subsection{Statistical Analysis}

Data analysis was carried out using Statistical Package for Social Sciences version 20.0 (SPSS, Statistics for Windows, IBM Corp, Armonk, NY, USA). The mean and standard deviation (SD) were used to express the data values. Statistical significance was determined using multiple comparisons between the three groups performed by a one-way ANOVA (analysis of variance) supplemented with the post hoc LSD test. Paired quantitative continuous data were compared using paired $t$ test. Receiver operating characteristic (ROC) curve was plotted for PC, MPV and PDW. Based on calculations of sensitivity and specificity for cut-off value of the three platelet indices, the area under curve (AUC) for each of them was determined. The largest AUC is associated with the best parameter (marker) for PE development [18]. $p<0.05$ was considered statistically significant. The strength of the association between changes in each one of the three studied platelet indices and MAP was estimated using the Pearson correlation coefficient $(r)$. The parameter with the most statistically significant correlation with MAP is the best marker of predicting severity of hypertension.

\section{Results}

During the study period, 2859 attendants met the inclusion criteria. However, only 2813 were included in the final analysis. Figure 1 represents the flow chart of recruitment.

Demographic and baseline characteristics of all participants are shown in Table 1. With the exception of gravidity which showed statistically significant difference between participants in all groups $(p=0.004)$, the three groups were similar in all characteristics that were recorded.

Table 2 shows comparisons between changes in PC, MPV and PDW in the three study groups. With the exception of few patients in the group of PE with severe features who developed thrombocytopenia, participants in all groups had a normal PC, but it showed continuous decrease over the course of pregnancy starting from 24 28 weeks gestation onwards. This change was more pronounced in PE without severe features than normotensive pregnancy and in PE with severe features than PE without severe features $(p=0.00)$. Changes in platelet 


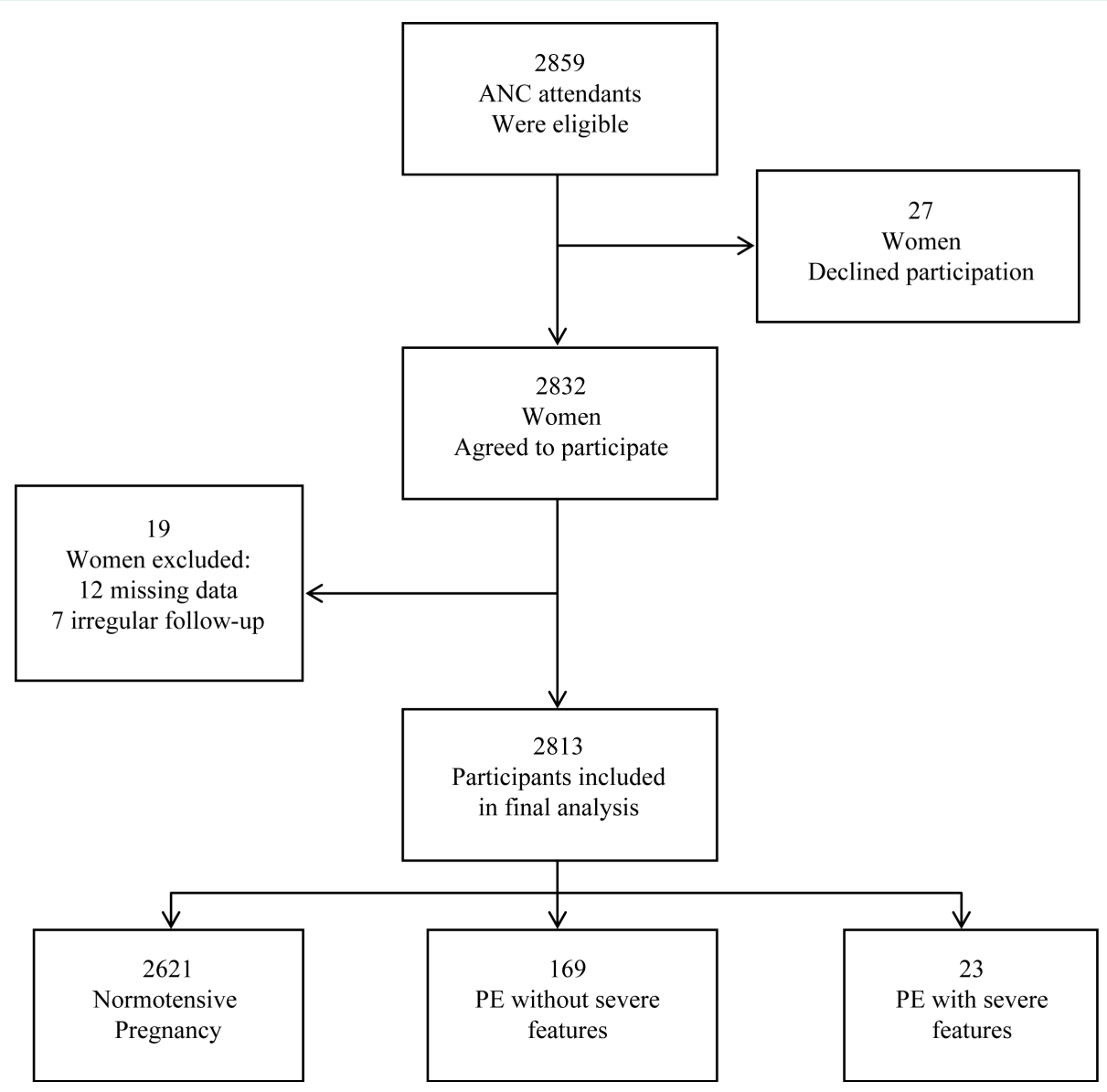

Figure 1. Flow chart of recruitment. ANC: Ante-natal clinic; PE: Preeclampsia.

Table 1. Demographic and baseline characteristics of all participants [data are presented as mean \pm standard deviation $( \pm \mathrm{SD})$, number $(\%)]$.

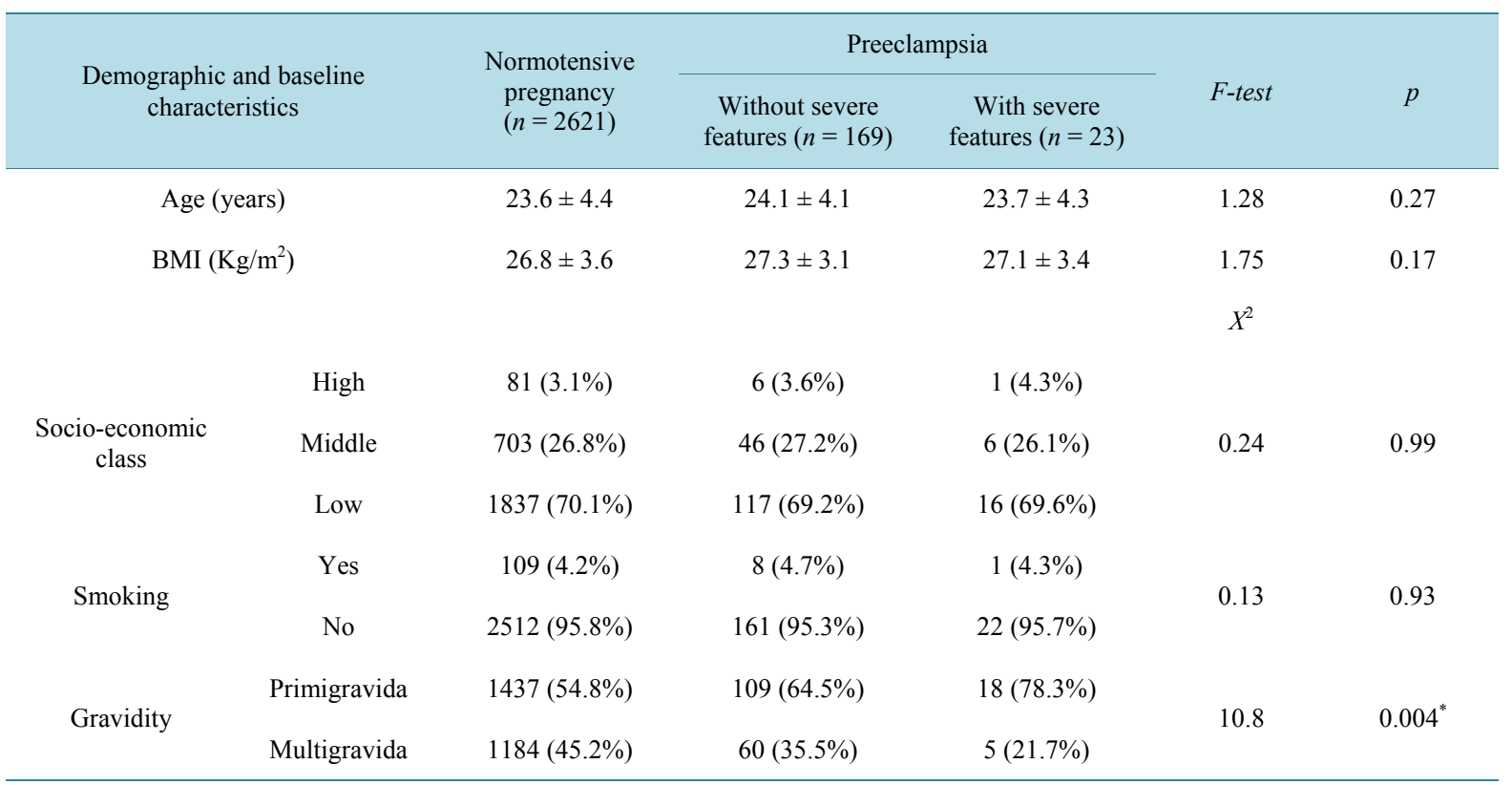

BMI: Body mass index. F-test: test used for analysis of variance (ANOVA). $X^{2}$ : Chi-squared test. 
Table 2. Comparisons between three platelet indices and mean arterial pressure in normotensive pregnancy, and preeclampsia [data are presented as mean \pm standard deviation $( \pm \mathrm{SD})]$.

\begin{tabular}{|c|c|c|c|c|c|c|}
\hline \multirow[b]{2}{*}{ Platelet indices } & \multirow[b]{2}{*}{ Gestational week } & \multirow{2}{*}{$\begin{array}{l}\text { Normotensive } \\
\text { pregnancy } \\
(n=2621)\end{array}$} & \multicolumn{2}{|c|}{ Preeclampsia } & \multirow[b]{2}{*}{ F-test } & \multirow[b]{2}{*}{$p$} \\
\hline & & & $\begin{array}{c}\text { Without severe } \\
\text { features }(n=169)\end{array}$ & $\begin{array}{c}\text { With severe } \\
\text { features }(n=23)\end{array}$ & & \\
\hline \multirow{2}{*}{$\mathrm{PC}\left(\times 10^{3} / \mathrm{mm}^{3}\right)$} & $24-28$ (Onset of change) & $262.6 \pm 49.08$ & $207 \pm 38.6$ & $177 \pm 17.9$ & 7.9 & $0.00^{* *}$ \\
\hline & $30-32$ (Onset of diagnosis) & $245.3 \pm 34.6$ & $191 \pm 40.3$ & $162 \pm 14.7$ & 8.1 & $0.00^{*}$ \\
\hline \multirow{2}{*}{ MPV (fL) } & $24-28$ (Onset of change) & $8.4 \pm 1.6$ & $10.8 \pm 1.7$ & $12.1 \pm 1.1$ & 5.17 & $0.003^{*}$ \\
\hline & $30-32$ (Onset of diagnosis) & $8.7 \pm 1.1$ & $11.2 \pm 2.3$ & $14.3 \pm 2.3$ & 5.64 & $0.002^{*}$ \\
\hline \multirow{2}{*}{ PDW (\%) } & $24-28$ (Onset of change) & $13.3 \pm 2.6$ & $24.4 \pm 3.6$ & $34.4 \pm 4.5$ & 24.3 & $0.00^{* *}$ \\
\hline & $30-32$ (Onset of diagnosis) & $15.4 \pm 1.7$ & $26.6 \pm 3.9$ & $36.9 \pm 6.8$ & 25.1 & $0.00^{* *}$ \\
\hline \multirow{2}{*}{ MAP } & $24-28$ (Onset of change) & $78.3 \pm 7.6$ & $95.2 \pm 6.6$ & $103.1 \pm 5.2$ & 6.9 & $0.00^{* *}$ \\
\hline & $30-32$ (Onset of diagnosis) & $85.4 \pm 4.6$ & $107.7 \pm 8.6$ & $129.7 \pm 7.8$ & 7.3 & $0.00^{* *}$ \\
\hline
\end{tabular}

PC: Platelet count; MPV: Mean platelet volume; PDW: Platelet distribution width; MAP: Mean arterial pressure; F-test: test used for analysis of variance (ANOVA).

Table 3. Comparison between the diagnostic values of platelet indices in all participants.

\begin{tabular}{cccccc}
\hline Platelet indices & Sensitivity (\%) & Specificity (\%) & cut-off & $\begin{array}{c}\text { AUC } \\
(95 \% \text { CI })\end{array}$ & $\begin{array}{c}\text { RR } \\
(95 \% \text { CI })\end{array}$ \\
\hline PC $\left(\times 10^{3} / \mathrm{mm}^{3}\right)$ & 81.5 & 78.3 & $<233$ & 0.171 & 4.22 \\
MPV (fL) & 92.6 & 87.0 & $>9.053-0.288)$ & $(1.87-9.56)$ \\
PDW (\%) & 96.3 & & & 0.940 & 8.41 \\
& & & & $(0.861-1.000)$ & $(2.88-24.92)$ \\
\end{tabular}

PC: Platelet count; MPV: Mean platelet volume; PDW: Platelet distribution width; AUC: Area under curve; RR: Relative risk; 95\% CI: 95\% confidence interval.

indices were predictive of PE development with onset of PE diagnosis at 30 - 32 weeks gestation. As such, these changes in women with PE predated development of disorder by $2-8$ weeks.

Compared to baseline values, MPV and PDW in participants in all groups showed continuous increase over the course of pregnancy starting from $24-28$ weeks gestation onwards. This change was more pronounced in PE without severe features than normotensive pregnancy and in PE with severe features than PE without severe features $(p=0.00)$.

Figure 2 shows the ROC curve of platelet indices in the two PE groups (with and without severe features) which identifies the optimal cut-off level for prediction of development of PE. PDW was seen to have the largest AUC (0.980 (95\% CI: 0.964 - 1.000), making it the best parameter (marker) for predicting PE development.

Figure 3 shows correlation between MAP ( $\mathrm{mmHg}$ ) and platelet indices. In women with PE (with and without severe features), PC was shown to have a negative correlation with MAP, whereas MPV and PDW showed positive correlations. These changes were also proportional to changes in MAP. Amongst the evaluated platelet indices, PDW was observed to have the most statistically significant correlation with $\mathrm{MAP}^{* *}(r=0.902, p=$ 0.000), making it the best marker for predicting severity of hypertension.

Table 3 shows comparisons between the diagnostic values of the selected platelet indices of participants in the three study groups. 


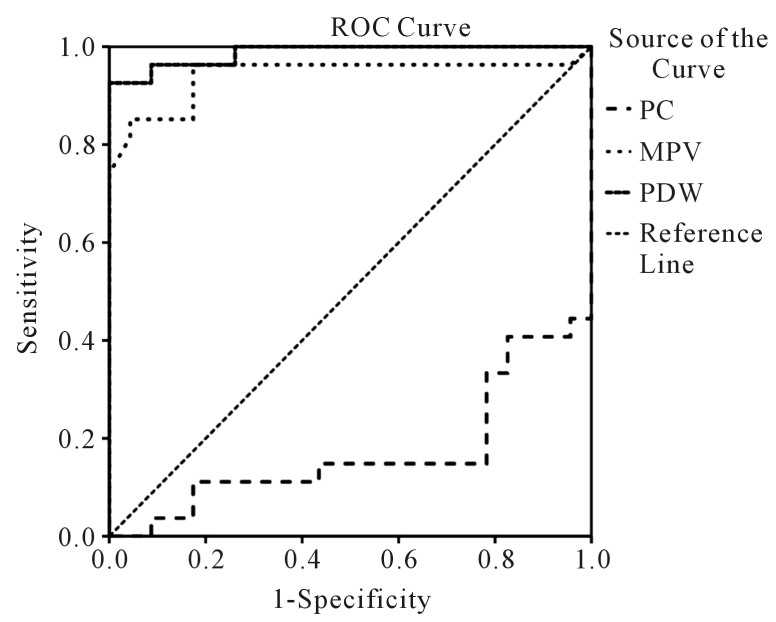

Diagonal segments are produced by ties

Figure 2. Receiver operating characteristic (ROC) curve of platelet indices in the preeclampsia (PE) groups (with and without severe features) at $24-28$ weeks gestation. The curve has the following area under the curve (AUC) values: Platelet count (PC): 0.171 (95\% CI: 0.053 - 0.288), Mean platelet volume (MPV): 0.940 (95\% CI: 0.861 - 1.000), and Platelet distribution width (PDW): 0.980 (95\% CI: 0.964 - 1.000).

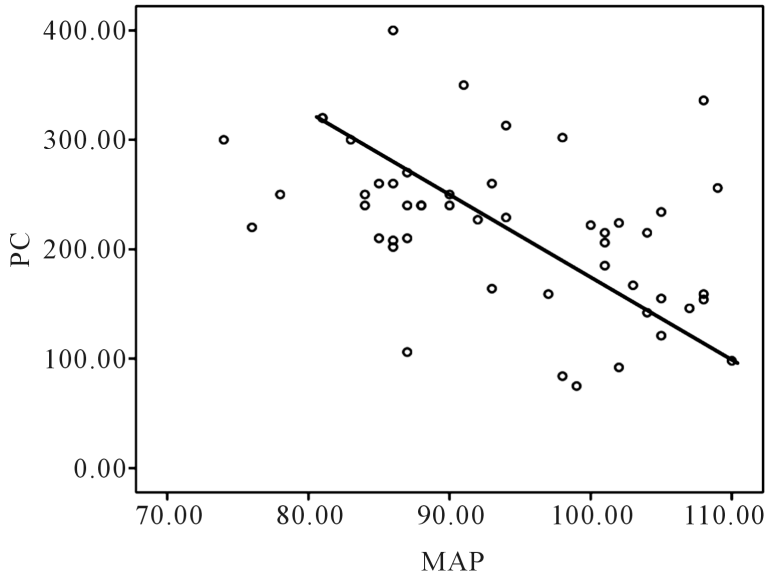

(a)

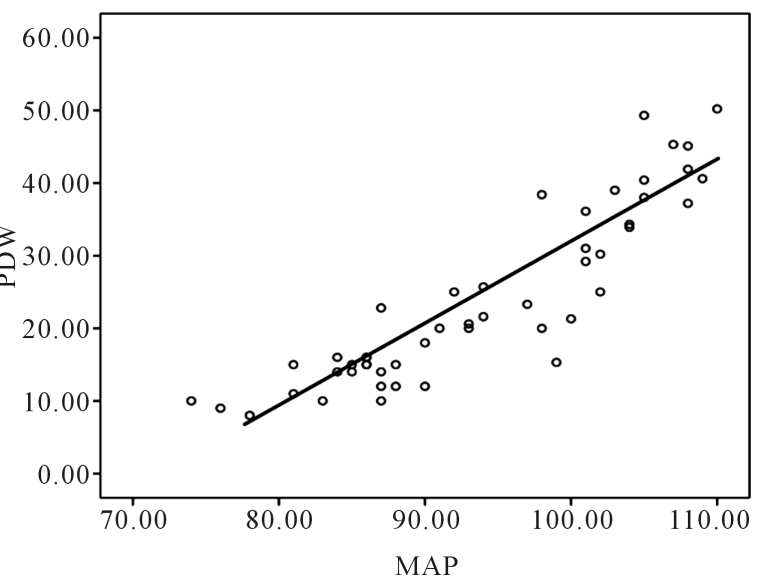

(c)

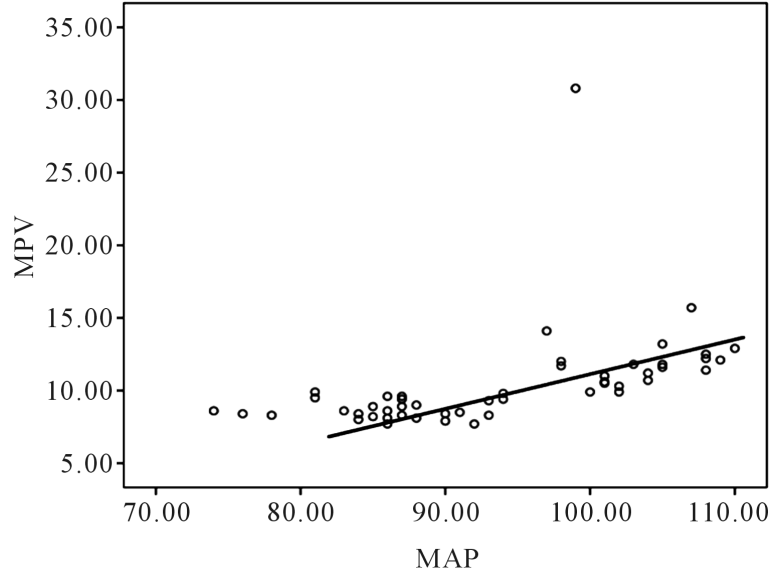

(b)

Figure 3. Correlation between the mean arterial pressure (MAP) $(\mathrm{mmHg})$ and platelet indices (Pearson rank correlation test). The correlation coefficient in each graph was: (a) Platelet count (PC) $r=-0.474^{* *}(p=0.001)$; (b) Mean platelet volume (MPV) $r=0.475^{* *}(p=0.000)$, and (c) Platelet distribution width (PDW) $r=0.902^{* *}(p=0.000)$. 


\section{Discussion}

Preeclampsia continues to be a major cause of maternal and fetal morbidity and mortality, as it is associated with a high risk of IUGR, preterm delivery, placental abruption, ...etc. [19]-[28]. Furthermore, there is a concern about the long-term consequences of PE, as women who experience this disorder represent a high-risk group for subsequent premature cardiovascular, cerebrovascular, peripheral arterial diseases and other chronic illnesses later in life [19] [22] [29]-[33]. Early onset PE, which requires delivery before 34 weeks gestation is the clinical form that largely contributes to these increased risks [19].

Changes in PC noted in this study are in agreement with those reported by other researchers [9] [34]-[36]. Inter-group analysis in this study revealed that changes in MPV values in PE-developing women (with and without severe features) were statistically more significant than normotensive pregnant women and this change was noted to start from the $24^{\text {th }}$ gestational week up to time of delivery $(p=0.003)$. In the two PE groups, onset of diagnosis was at $30-32$ weeks gestation, and significant MPV increase was noted to precede development of PE by approximately $2-8$ weeks.

In this study, ROC curve analysis of changes of MPV value at $24-28$ weeks gestation, produced a MPV > $9.5 \mathrm{fL}$ as optimal cut-off for the prediction of PE development. This cut-off level had an AUC of 0.940 (95\% CI: $0.861-1.000$ ), a sensitivity of $92.6 \%$ and a specificity of $87.0 \%$ for prediction of PE development. Women with MPV $>9.5 \mathrm{fL}$ at $24-28$ weeks gestation were more than eight times at risk of PE development (RR: 8.41; $95 \%$ CI: 2.88 - 24.92). Also, in women with PE, MPV was noted to have a positive correlation with MAP, and MPV increase was proportional to the increase in MAP $(r=0.475 ; p=0.000)$; making MPV a possible modest marker for prediction of severity of hypertension. This is in agreement with what was concluded by other investigators [9] [33].

Validity of changes in MPV in predicting PE has been extensively investigated. Dundar et al., in a previous large longitudinal study suggested that increase of MPV may precede development of PE symptoms by approximately 4.6 weeks (range $2.8-5.9$ weeks) [13]. They suggested that sensitivity and specificity of MPV value at $24-28$ gestational weeks with a cut-off value of $8.5 \mathrm{fL}$ for predicting PE development were $78 \%$ and $86 \%$, respectively. Accordingly, they concluded that women with MPV $>8.5 \mathrm{fL}$ at $24-28$ weeks were almost three times at risk of PE development (OR: 2.83). In the light of these results, they suggested that serial MPV monitoring may assist obstetricians and other healthcare givers in predicting development of PE. Similarly, Järemo et al. confirmed that women with high MPV in the second trimester of pregnancy had an increased risk of developing PE later on during pregnancy [14]. They suggested that MPV stands as a marker for prediction of PE development. Furthermore, Gioia et al. proposed that a MPV cut-off value $\geq 10 \mathrm{fL}$ may be considered as a further significant parameter that is able to predict unfavourable neonatal outcome in women affected by altered uterine artery Doppler velocimetry [37].

In the two PE groups in this study, onset of diagnosis using changes in PDW started at 30 - 32 weeks gestation, and significant PDW increase was noted to precede development of PE by approximately $2-8$ weeks. Our results are in agreement with those of previous studies [9] [35] [36].

In this study, ROC curve analysis of changes of PDW values at $24-28$ weeks gestation produced a PDW> 19.9 as optimal cut-off for the prediction of PE development. This cut-off level had an AUC of 0.980 (95\% CI: $0.964-1.000$ ), a sensitivity of $96.3 \%$ and a specificity of $91.3 \%$ for prediction of PE development. Women with PDW > 19.9 at $24-28$ weeks gestation were more than 13 times at risk of PE development (RR: 13.36; 95\% CI: 3.5 - 50.96). Also, in women with PE, PDW was noted to have a positive correlation with MAP, and PDW increase was proportional to the increase in MAP $(r=-0.902 ; p=0.000)$; making PDW the best potential marker for prediction of severity of hypertension.

However, Yang et al. identified a PDW value of $>13.5$ as the optimal cut-off level for the prediction of PE severity [9]. They noted that this value had an AUC of 0.74 , a sensitivity of $72 \%$ and a specificity of $71 \%$. Also, they reported that only PDW had a statistically significant correlation with MAP $(r=0.231, p=0.011)$ and, accordingly, they concluded that PDW was the best PE marker. Similarly, Freitas et al., identified a PDW value of $>18.3$ as the optimal cut-off level for the prediction of PE [35]. They noted that this value had an AUC of 0.77 (95\% CI: $0.66-0.85$ ), a sensitivity of $55.17 \%$ and a specificity of $86.21 \%$. Also, they reported that only PDW had a statistically significant correlation with MAP $(r=0.231, p=0.011)$.

Conflicting results have been published regarding changes in platelet indices in normotensive pregnancies and PE. Some investigators found no difference in values of these indices between PE and controls [15] [38], whereas 
others demonstrated lower PC and higher MPV and PDW in PE [9] [13] [14] [16]. It is suggested that the major reason for the inconsistency between these studies is probably the method of measurement of these platelet indices. Measurements performed in EDTA change in a time-dependent manner. Also, different systems used in measurement can yield different results, and this difference may reach up to $40 \%$ [15].

The strengths of this study are worth mentioning. All blood samples for CBC assessment were processed within two hours after venepuncture. Assessments were carried out using the same anticoagulant and the same automated counter. The large numbers of recruited participants as well as enrolment of women early in pregnancy give further credit to our conclusions.

\section{Conclusion}

This study provides evidence that PC decreases while MPV and PDW increase as pregnancy advances, and these changes are more pronounced in PE than normotensive pregnancy. Changes in platelet indices predate development of PE by 2 - 8 weeks and are proportional to the progress of this disorder. The selected platelet indices, especially PDW, have the potential to be utilized as markers for not only prediction of PE development but also severity of hypertension.

\section{Acknowledgements}

The authors would like to thank junior medical, midwifery and nursing staff of Obstetrics and Gynecology Department at ZUH for their contribution to collection of the data of this study.

\section{Declaration of Interest}

The authors report no conflicts of interest.

\section{Role of the Funding Source}

The authors alone were responsible for funding this work. They have not received any grants, financial support or any other source of funding.

\section{References}

[1] Ananth, C.V., Keyes, K.M. and Wapner, R.J. (2013) Preeclampsia Rates in the United States, 1980-2010: Age-PeriodCohort Analysis. BMJ, 347, f6564. http://dx.doi.org/10.1136/bmj.f6564

[2] (2000) Report of the National High Blood Pressure Education Program Working Group on High Blood Pressure in Pregnancy. American Journal of Obstetrics and Gynecology, 183, S1-S22.

[3] Ness, R.B. and Sibai, B.M. (2006) Shared and Disparate Components of the Pathophysiologies of Fetal Growth Restriction and Preeclampsia. American Journal of Obstetrics and Gynecology, 195, 40-49. http://dx.doi.org/10.1016/j.ajog.2005.07.049

[4] Sibai, B.M. (2011) Evaluation and Management of Severe Preeclampsia before 34 Weeks' Gestation. American Journal of Obstetrics and Gynecology, 205, 191-198. http://dx.doi.org/10.1016/j.ajog.2011.07.017

[5] Sibai, B.M., Dekker, G. and Kupferminc, M. (2005) Preeclampsia. The Lancet, 365, 785-799. http://dx.doi.org/10.1016/S0140-6736(05)71003-5

[6] (2013) Report of the ACOG Task Force on Hypertension in Pregnancy. Hypertension in Pregnancy. Obstetrics \& Gynecology, 122, 1122-1131.

[7] Bujold, E., Roberge, S., Lacasse, Y., et al. (2010) Prevention of Preeclampsia and Intrauterine Growth Restriction with Aspirin Started in Early Pregnancy: A Meta-Analysis. Obstetrics \& Gynecology, 116, 402-414. http://dx.doi.org/10.1097/aog.0b013e3181e9322a

[8] Sibai, B.M. (2003) Diagnosis and Management of Gestational Hypertension and Preeclampsia. Obstetrics \& Gynecology, 102, 181-192. http://dx.doi.org/10.1016/S0029-7844(03)00475-7

[9] Yang, S.W., Cho, S.H., Kwon, H.S., et al. (2014) Significance of the Platelet Distribution Width as a Severity Marker for the Development of Preeclampsia. European Journal of Obstetrics \& Gynecology and Reproductive Biology, 175, 107-111. http://dx.doi.org/10.1016/j.ejogrb.2013.12.036

[10] Scazzocchio, E., Figueras, F., Crispi, F., et al. (2013) Performance of a First-Rimester Screening of Preeclampsia in a Routine Care Low-Risk Setting. American Journal of Obstetrics and Gynecology, 208, 203.e1-203.e10. 
[11] Akolekar, R., Syngelaki, A., Sarquis, R., et al. (2011) Prediction of Early, Intermediate and Late Preeclampsia from Maternal Factors, Biophysical and Biochemical Markers at 11-13 Weeks. Prenatal Diagnosis, 31, 66-74. http://dx.doi.org/10.1002/pd.2660

[12] Scazzocchio, E. and Figueras, F. (2011) Contemporary Prediction of Preeclampsia. Current Opinion in Obstetrics and Gynecology, 23, 65-71. http://dx.doi.org/10.1097/gco.0b013e328344579c

[13] Dundar, O., Yoruk, P., Tutuncu, L., et al. (2008) Longitudinal Study of Platelet Size Changes in Gestation and Predictive Power of Elevated MPV in Development of Preeclampsia. Prenatal Diagnosis, 28, 1052-1056. http://dx.doi.org/10.1002/pd.2126

[14] Järemo, P., Lindahl, T.L., Lennmarken, C., et al. (2000) The Use of Platelet Density and Volume Measurements to Estimate the Severity of Preeclampsia. European Journal of Clinical Investigation, 30, 1113-1118. http://dx.doi.org/10.1046/j.1365-2362.2000.00753.x

[15] Ceyhan, T., Beyan, C., Baser, I., et al. (2006) The Effect of Preeclampsia on Complete Blood Count, Platelet Count and Mean Platelet Volume. Annals of Hematology, 85, 320-322. http://dx.doi.org/10.1007/s00277-006-0091-7

[16] Vagdatli, E., Gounari, E., Lazaridou, E., et al. (2010) Platelet Distribution Width: A Simple, Practical and Specific Marker of Activation of Coagulation. Hippokratia, 14, 28-32.

[17] Brown, M.A., Lindheimer, M.D., de Swiet, M., et al. (2001) The Classification and Diagnosis of the Hypertensive Disorders of Pregnancy: Statement from the International Society for the Study of Hypertension in Pregnancy (ISSHP). Hypertension in Pregnancy, 20, 9-14. http://dx.doi.org/10.3109/10641950109152635

[18] Martinez, E.Z., Louzada-Neto, F. and Pereira, B.B. (2003) A curva ROC para testes diagnósticos. Cadernos Saúde Coletiva, 11, 7-31.

[19] Simeone, S., Lojo, C., Garcia-Esteve, L., et al. (2015) Psychological Impact of First-Trimester Prevention for Preeclampsia on Anxiety. Prenatal Diagnosis, 35, 60-64. http://dx.doi.org/10.1002/pd.4485

[20] Trogstad, L., Magnus, P. and Stoltenberg, C. (2011) Preeclampsia: Risk Factors and Causal Models. Best Practice \& Research Clinical Obstetrics \& Gynaecology, 30, 1-14.

[21] Hernandez-Diaz, S., Toh, S. and Cnattingius, S. (2009) Risk of Preeclampsia in First and Subsequent Pregnancies: Prospective Cohort Study. BMJ, 338, b2255. http://dx.doi.org/10.1136/bmj.b2255

[22] McDonald, S.D., Malinowski, A., Zhou, Q., et al. (2008) Cardio-Vascular Sequelae of Preeclampsia/Eclampsia: A Systematic Review and Meta-Analyses. American Heart Journal, 156, 918-930. http://dx.doi.org/10.1016/j.ahj.2008.06.042

[23] Roberts, J.M. and Cooper, D.W. (2001) Pathogenesis and Genetics of Preeclampsia. The Lancet, 357, 53-56.

[24] Tanaka, M., Jaamaa, G., Kaiser, M., et al. (2007) Racial Disparity in Hypertensive Disorders of Pregnancy in New York State: A 10-Year Longitudinal Population-Based Study. American Journal of Public Health, 97, 163-170. http://dx.doi.org/10.2105/AJPH.2005.068577

[25] Ananth, C.V. and Basso, O. (2010) Impact of Pregnancy-Induced Hypertension on Stillbirth and Neonatal Mortality. Epidemiology, 21, 118-123. http://dx.doi.org/10.1097/EDE.0b013e3181c297af

[26] Goldenberg, R.L., Culhane, J.F., Iams, J.D., et al. (2008) Epidemiology and Causes of Preterm Birth. The Lancet, 371, 75-84. http://dx.doi.org/10.1016/S0140-6736(08)60074-4

[27] Zhang, J., Troendle, J.F. and Levine, R.J. (2001) Risks of Hypertensive Disorders in the Second Pregnancy. Paediatric and Perinatal Epidemiology, 15, 226-231. http://dx.doi.org/10.1046/j.1365-3016.2001.00347.x

[28] Conde-Agudelo, A., Belizan, J.M. and Diaz-Rossello, J.L. (2000) Epidemiology of Fetal Death in Latin America. Acta Obstetricia et Gynecologica Scandinavica, 79, 371-378. http://dx.doi.org/10.1080/j.1600-0412.2000.079005371.x

[29] Ray, J.G., Vermeulen, M.J., Schull, M.J., et al. (2005) Cardiovascular Health after Maternal Placental Syndromes (CHAMPS): Population-Based Retrospective Cohort Study. The Lancet, 366, 1797-1803. http://dx.doi.org/10.1016/S0140-6736(05)67726-4

[30] Irgens, H.U., Reisaeter, L., Irgens, L.M., et al. (2001) Long Term Mortality of Mothers and Fathers after Preeclampsia: Population Based Cohort Study. BMJ, 323, 1213-1217. http://dx.doi.org/10.1136/bmj.323.7323.1213

[31] Guzin, K., Tomruk, S., Tuncay, Y.A., et al. (2005) The Relation of Increased Uterine Artery Blood Flow Resistance and Impaired Trophoblast Invasion in Pre-Eclamptic Pregnancies. Archives of Gynecology and Obstetrics, 272, 283288. http://dx.doi.org/10.1007/s00404-005-0005-2

[32] Kuscu, N.K., Kurhan, Z., Yildirim, Y., et al. (2003) Detection of Endothelial Dysfunction in Preeclamptic Patients by Using Colour Doppler Sonography. Archives of Gynecology and Obstetrics, 268, 113-116.

[33] Missfelder-Lobos, H., Teran, E., Lees, C., et al. (2002) Platelet Changes and Subsequent Development of Preeclampsia and Fetal Growth Restriction in Women with Abnormal Uterine Artery Doppler Screening. Ultrasound in Obstetrics and Gynecology, 19, 443-448. http://dx.doi.org/10.1046/j.1469-0705.2002.00672.x 
[34] Vijaya, C., Lekha, M.B., Shetty, A., et al. (2014) Evaluation of Platelet Counts and Platelet Indices and Their Significant Role in Preeclampsia and Eclampsia. Journal of Evolution of Medical and Dental Sciences, 3, 3216-3219. http://dx.doi.org/10.14260/jemds/2014/2269

[35] Freitas, L.G., Alpoim, P.N., Komatsuzaki, F., et al. (2013) Preeclampsia: Are Platelet Count and Indices Useful for Its Prognostic? Hematology, 18, 360-364. http://dx.doi.org/10.1179/1607845413Y.0000000098

[36] Dadhich, S., Agrawal, S., Soni, M., et al. (2012) Predictive Value of Platelet Indices in Development of Preeclampsia. Journal of SAFOG with DVD, 4, 17-21. http://dx.doi.org/10.5005/jp-journals-10006-1164

[37] Gioia, S., Piazze, J., Anceschi, M.M., et al. (2007) Mean Platelet Volume: Association with Adverse Neonatal Outcome. Platelets, 18, 284-288. http://dx.doi.org/10.1080/09537100601078448

[38] Makuyana, D., Mahomed, K., Shukusho, F.D., et al. (2002) Liver and Kidney Function Tests in Normal and PreEclamptic Gestation-A Comparison with Non-Gestational Reference Values. Central African Journal of Medicine, 48 , 55-59. 